\title{
Quantitation of Lipids by Charring on Thin-Layer Plates and Scintillation Quenching: Application to Ceramide Determination
}

\author{
Ramasamy Selvam and Norman S. Radin \\ Mental Health Research Institute (Department of Psychiatry) and Department of Biological Chemistry, \\ University of Michigan, Ann Arbor, Michigan 48109
}

Received September 15,1980

\begin{abstract}
A method is described for the quantitative determination of lipids after separation by thinlayer chromatography: the lipid spots are visualized with a charring spray and the darkness of the spots is determined by measuring the quenching produced when the powder is suspended in a scintillation counting gel containing a radioactive sample. A standard curve produced with 2 to $20 \mu \mathrm{g}$ of ceramide was linear, so only two standard spots (in duplicate) are needed on each thin-layer plate. Using the vertical sample streak technique, one can analyze three unknown samples (in duplicate) on each plate. This technique allows one to use the quantitative thin-layer approach without purchasing a special apparatus for the purpose. As an example of the method, we analyzed tissues for ceramide content, after first removing much polar lipid by a liquid/liquid partitioning technique. This is the most convenient method available for determining this minor, but widely distributed lipid.
\end{abstract}

The quantitative determination of many substances can be accomplished by separating them by tlc, ${ }^{1}$ then revealing their presence with a chromogenic reaction and measuring the amount of material with an optical scanning device. Snyder and Moehl (1) and Noble et al. (2) have pointed out that a liquid scintillation counter can be used as a photometer for measuring the color intensity of a solution by suspending a light source in a counting vial containing the unknown solution. The light source used was a combination of radioactive material and a scintillator which could be calibrated and reused after washing. The lightabsorbing sample produced optical quenching, which could be measured through the observed lowered activity of the source. Shand and Noble (3) adapted this procedure to the analysis of charred tlc spots by

\footnotetext{
' Abbreviation used: tlc, thin-layer chromatography.
}

suspending the tlc powder in a gelled scintillation solvent and producing light flashes in the gel with the external radioactive source used as an external standard in many scintillation counters. The observed quenching ratio in two specific counting channels was used as a measure of the amount of sample in the tlc powder.

The use of a scintillation counter instead of a scanner may seem wasteful, but the former is more widely available and may be intrinsically capable of yielding higher reproducibility. If a counter is not being used in the usual mode full time, this approach obviates or tests the need to buy an additional expensive device.

The Shand and Noble procedure called for relatively large amounts of lipid and utilized a single large tlc plate for each sample, which was applied as a long streak. The reported variability in the measured amounts seemed excessively high, possibly because their calibration curve was curved and be- 
cause of interplate variations. The use of a single plate for each sample made the procedure quite expensive. We report here modifications of the procedure which result in a method allowing one to apply 9 or 10 samples to one plate, with somewhat smaller amounts of the component of interest $(2$ to $20 \mu \mathrm{g}$ ). Since the calibration curve is now linear, only two standards need be applied to each plate. The method should be applicable to a wide variety of compounds.

The method was applied to the problem of determining ceramide in tissues. This lipid is a key intermediate in the biosynthesis and degradation of all the sphingolipids and knowledge of its concentration in tissues should be useful. Few such determinations have been made, in part because the concentration is low, in part because no specific color reaction has been devised for sphingolipids. Previously reported procedures involved high-performance liquid chromatography of the benzoate ester $(4,5)$ or ketonic oxidation product (6), gas-liquid chromatography $(7,8)$, direct weighing of the isolated lipid (9), determination of a degradation product, such as fatty acid esters or sphingoid base $(10-13)$, or visually estimated tlc spots (14). Many of these papers treated the quantitative aspects of the analytical method in an incomplete manner. All of the procedures involved many steps and are inherently subject to relatively large experimental errors and losses. It seemed likely to us that tlc and a quantitative charring procedure would be more practical. However, the low concentration of ceramide in lipids makes it necessary to first remove as much as possible of the more polar lipids in tissue extracts, to prevent overloading of the plates. This can be accomplished in one step by column chromatography but the problem of clogging, due to insoluble polar components, makes it necessary to introduce an extra step. We found that liquid/liquid partitioning readily removes much of the polar components and is more convenient when many samples must be run.

\section{MATERIALS}

Glass-based tlc plates, $20 \times 20 \mathrm{~cm}$, coated with silica gel $600.25 \mathrm{~mm}$ thick, were obtained from EM Laboratories (No. 5763, E. Merck) and used without reactivation or washing. Solvents, redistilled, were from Burdick \& Jackson Laboratories. Stearoyl sphingosine, used as a standard, was prepared in this laboratory $(15,16)$ from sphingosine and $\left[{ }^{14} \mathrm{C}\right]$ stearic acid or unlabeled stearic acid. The labeled ceramide and fatty acid were repurified by partitioning between hexane, isopropanol, and alkaline or acid water. A tritium-labeled lipid (cholesterol) was used as internal standard. Scraped tlc powders were counted in Aquasol, a water-miscible scintillation fluid from New England Nuclear.

\section{METHODS}

Lipid extraction. The method used was a slight modification of our previous one (17). To $1 \mathrm{~g}$ of liver in a test tube was added $18 \mathrm{ml}$ of hexane-isopropanol $3: 2$, the mixture was homogenized with a Tekmar Tissumizer (Ultra-Turrax) for $1 \mathrm{~min}$, and the suspension was filtered through a Buchner funnel fitted with a sintered glass disk and a ball joint for application of pressure. The homogenizer, funnel, and tissue residue were washed with $2 \times 3-\mathrm{ml}$ portions of solvent; each time the residue was resuspended and allowed to soak 2 min before applying pressure.

Aliquots of the extract $(6 \mathrm{ml}$, equivalent to $245 \mathrm{mg}$ of tissue) were transferred to $16 \times 150$-mm screw-cap test tubes. For assessing the recovery of $\left[{ }^{14} \mathrm{C}\right]$ ceramide or $\left[{ }^{14} \mathrm{C}\right]$ stearic acid or unlabeled ceramide, the lipids were predried in the tubes before adding the extract. The extracts were then vortexed for $1 \mathrm{~min}$ with $1.8 \mathrm{ml}$ of $3.5 \%$ $\mathrm{Na}_{4} \mathrm{~B}_{2} \mathrm{O}_{7} \cdot 10 \mathrm{H}_{2} \mathrm{O}$ containing $0.01 \%$ cresol red. 
The upper liquid phase, which contained the less-polar lipids, separated readily without centrifugation. A white precipitate of borate could be seen. Most of the upper layer was transferred to a $15-\mathrm{ml}$ conical centrifuge tube with a suction transferring system, in which 1/16-in. stainless-steel tubing contacted the liquid. This was rinsed with solvent after each sample.

The lower layer was extracted with $5 \mathrm{ml}$ of hexane-isopropanol $7: 2$ by vortexing again and the upper layer was pooled with the first upper layer, using the same transfer device. This hexane-rich extract was then evaporated with a stream of nitrogen to $<1 \mathrm{ml}$ (using an N-EVAP, Organomation Assoc., Northborough, Mass.). The solution was transferred with a Pasteur pipet, using $2 \times 0.5-\mathrm{ml}$ rinses, either to a $2-$ $\mathrm{ml}$ screw-cap conical tube for tic or to a 7 $\mathrm{ml}$ polyethylene scintillation counting vial ("Minivial") for radioactivity measurement. In the latter case, the solvent was removed by evaporation and the residue was dissolved in $0.3 \mathrm{ml}$ water and $5 \mathrm{ml}$ of a Triton-xylene scintillation mixture (18).

For the tlc step, the solvent was removed with nitrogen and the residue was dissolved in chloroform-methanol $2: 1$, using a volume expected to yield a ceramide concentration of 0.2 to $0.4 \mu \mathrm{g} / \mu \mathrm{l}$. Typically we used $125 \mu \mathrm{l}$ in the case of mouse or rat liver, $500 \mu \mathrm{l}$ in the case of calf liver.

Thin-layer chromatography. The samples were applied to the plate by the vertical streak technique, without the use of an air blower. Duplicate aliquots of the lipid samples $(40 \mu \mathrm{l})$ were applied from a $50-\mu \mathrm{l}$ microsyringe as a $1.5-\mathrm{cm}$ band, between two pencil lines drawn 1 and $2.5 \mathrm{~cm}$ from, and parallel to, the bottom edge of the tlc plate. The sample bands were spaced about $1.75 \mathrm{~cm}$ apart, which allowed application of 10 samples with a bit of extra space at the two sides. To minimize the effect of solution evaporation, the sample tube was kept in ice while the syringe was loaded and applied to the tlc plate.
Two standard solutions were applied also, in duplicate. One contained $4 \mu \mathrm{g}$ and the other, $12 \mu \mathrm{g}$ of stearoyl sphingosine, both in $40 \mu$ l of chloroform-methanol $2: 1$. The two standards were streaked at the left side of the plate, three unknown streaks were applied in the adjacent positions, then the right half of the plate was loaded similarly with the duplicate samples.

The plates were chromatographed with chloroform-methanol-water $\quad 60: 35: 8$, which was allowed to rise to a third pencil line drawn $3.5 \mathrm{~cm}$ from the bottom. This moved the less-polar lipids to the solvent front in the form of a narrow band while leaving the more polar lipids as a vertical streak. After air drying for $15 \mathrm{~min}$, the plate was developed with chloroform-methanol -acetic acid 90:2:8 (19), which was run up to a groove scraped into the silica gel at a point $1.5 \mathrm{~cm}$ from the top.

After $45 \mathrm{~min}$ of air drying and $45 \mathrm{~min}$ of drying in a $37^{\circ} \mathrm{C}$ oven, the plates were sprayed until translucent with cupric acetate-phosphoric acid reagent (20) and heated in a gravity convection oven to make the spots visible. This charring spray is relatively insensitive to variations in lipid structure. Care was taken to make the spray uniform; after spraying, the plate was left in a horizontal position for several minutes to let gravity equilibrate the distribution of the liquid. The plate was placed in the cold oven onto a small insulating board centered on the wire shelf near one side wall, to maximize uniformity of exposure to hot air and radiant heat, then the heat was turned on, and the plate was left for $15 \mathrm{~min}$ after the oven reached $150^{\circ} \mathrm{C}$.

After the plate cooled, a pencil and ruler were used to divide the plate at the ceramide level into rectangles of equal area (typically $15 \mathrm{~mm}$ wide, $12 \mathrm{~mm}$ high) with some background silica gel in each rectangle. A broken razor blade (single edge) was used to detach one ceramide spot from the glass and the powder was transferred to a counting vial with a simplified form of the collec- 
TABLE 1

EFFect of Sodium Borate Concentration on THE EXTRACTION OF CERAMIde and FATTY ACID FROM LIVER LIPIDS ${ }^{a}$

\begin{tabular}{cccc}
\hline $\begin{array}{c}\text { Concentration } \\
\text { of borax } \\
\text { solution } \\
(\%, w / v)\end{array}$ & $\begin{array}{c}\text { Yield of } \\
{\left[{ }^{14} \mathrm{C}\right] \text {-ceramide }} \\
(\%)\end{array}$ & $\begin{array}{c}\text { Yield of } \\
{ }^{\text {19C-fatty }} \\
\text { acid }(\%)\end{array}$ & $\begin{array}{c}\text { Concentration of } \\
\text { ceramide in liver } \\
(\mu \mathrm{g} / \mathrm{g} \text { wet tissue) }\end{array}$ \\
\hline 1 & 88.6 & 7.0 & 133 \\
2 & 88.5 & 6.4 & 150 \\
3 & 87.7 & 6.8 & 147 \\
3.5 & 88.9 & 6.3 & 143 \\
4 & 88.5 & 6.6 & 144 \\
5 & 87.2 & 6.4 & 145 \\
\hline
\end{tabular}

a A hexane-isopropanol extract was prepared, containing labeled stearoyl sphingosine or stearic acid, and 6-ml portions were extracted with $1.8 \mathrm{ml}$ of aqueous borax. The radioactivity in the upper phase was measured and compared with the amount of labeled material added. In a third set of extracts, the content of ceramide in the upper layer was measured by the tlc/charring technique.

tor described by Platt (21). This consisted of a glass tube ( $5 \mathrm{~mm} \mathrm{o.d.,} 7 \mathrm{~cm}$ long) attached by a short plastic tube to one part of a small polyethylene "Quick Disconnect" tubing connector. The other part of the tubing connector was connected to a vacuum source, adjusted with a leak and needle valve to yield a weak suction. A 6-mm disk of glass fiber filter material (Gelman Instrument, Ann Arbor, Type A, $1 \mu \mathrm{m}$ porosity, cut from a larger disk with a cork borer) was sucked onto the open end of the glass tube, then brought into contact with the silica gel scrapings. The disk and adhering powder were allowed to fall into the Minivial by disconnecting the Quick Disconnect. It was necessary to use two filter disks to complete the transfer of the powder. This method avoided loss of powder by contact with tubes, filters, etc.

To each vial was added $0.8 \mathrm{ml}$ of water and $2 \mathrm{ml}$ of Aquasol scintillation fluid containing about $4300 \mathrm{cpm}$ of $\left[{ }^{3} \mathrm{H}\right]$ cholesterol. The capped vials were then placed in a polypropylene rack and immersed partially in an ultrasonic bath containing water warmed to $50^{\circ} \mathrm{C}$. After $2 \mathrm{~min}$ of sonication, the rack was removed from the bath and vials were removed in pairs and vortexed $10 \mathrm{~s}$ while cooling in air. When all vials had been vortexed, the agitation was repeated for two additional 10 -s periods. While the vials were still warm, the mixing produced a thin milky suspension of the silica gel, which then changed to a fairly firm, clearer gelled suspension. Each sample was then counted for $10 \mathrm{~min}$ in a Beckman Model LS 8000 counter. When extra counting time was available, the vials were counted a second time to improve the precision of the measurement.

\section{RESULTS AND DISCUSSION}

\section{The Extraction/Partition Step}

The efficacy of hexane-isopropanol for the extraction of most lipid classes has already been demonstrated, as well as the advantages of using a solvent system that is economical, relatively nontoxic, and chemically stable (17). Additionally, the system brings relatively little protein into the extract; protein can complicate subsequent purification steps. Tests with various aqueous solutions for preferential partitioning of ceramide, monitored by tlc, showed that an alkaline solution, made from $\mathrm{Na}$ borate, yielded an upper, hexane-rich phase that contained almost all the lipids of low polarity. The free fatty acids seemed to be entirely in the lower layer, due to the tendency of hexane solutions to repel sodium salts. This contrasts with chloroformmethanol-water partitions, which contain the soaps in the chloroform-rich layer. The volume of borate solution $(0.3 \mathrm{ml} / \mathrm{ml}$ extract) was chosen to yield upper and lower layers of about equal volume. Because the two layers are rather colorless, the inclusion of cresol red gives a very useful demarcation of the layers and minimizes accidental carryover of the lower layer into the upper.

A study with differing borate concentrations (Table 1) showed that a single partitioning step brought $89 \%$ of added $\left[{ }^{14} \mathrm{C}\right]$ ceramide into the upper layer, and left a little under $94 \%$ of $\left[{ }^{14} \mathrm{C}\right]$ stearic acid in the 
lower layer. While the radioactivity measurements seemed to show that any concentration of borate between 1 and $5 \%$ gave similar and reasonable results, analysis of the upper layer by the tlc charring method suggested that $1 \%$ borate was too low a concentration. Since the lower layer contained some of the ceramide, a second partitioning was carried out; this brought the recovery of added $\left[{ }^{14} \mathrm{C}\right]$ ceramide to $94 \%$. The final values were therefore corrected by dividing by 0.94 . The weight of lipid in the pooled upper layers comprised $60 \%$ of the total liver lipid weight.

A second type of recovery test was made by adding a known weight of unlabeled ceramide $(16 \mu \mathrm{g})$ to several $6-\mathrm{ml}$ aliquots of a mouse liver extract. Three unsupplemented aliquots, analyzed by the described procedure, were found to contain 25.1, 26.2, and $27.5 \mu \mathrm{g}$ and three supplemented aliquots were found to contain $41.9,43.5$, and $43.9 \mu \mathrm{g}$. The aliquots were analyzed in duplicates, with a pair of controls and a pair of supplemented spots on one plate. Using the data for each plate, we found the recoveries to be 105,108 , and $103 \%$, respectively.

\section{The thin-layer chromatography Step}

The lipid spot pattern obtained by tlc showed (Fig. 1) that the ceramide spot was clearly defined and satisfactorily separated from nearby spots. Although there is much more cholesterol than ceramide in tissues (roughly 80 times, in mouse liver) there was no sign of interference by the former. It has long been known that silica gel tlc plates can accomodate quite high weights of the low-polarity lipids. Only small amounts of free fatty acid and monoacyl glycerol could be seen. 1-O-Alkyl glycerol (batyl alcohol used as standard) could not be detected, although relatively large amounts could be seen in liver lipids after alkali-catalyzed methanolysis. Evidently borate solutions, despite their high $\mathrm{pH}$, do not cause breakdown of the low-polarity lipid esters. The

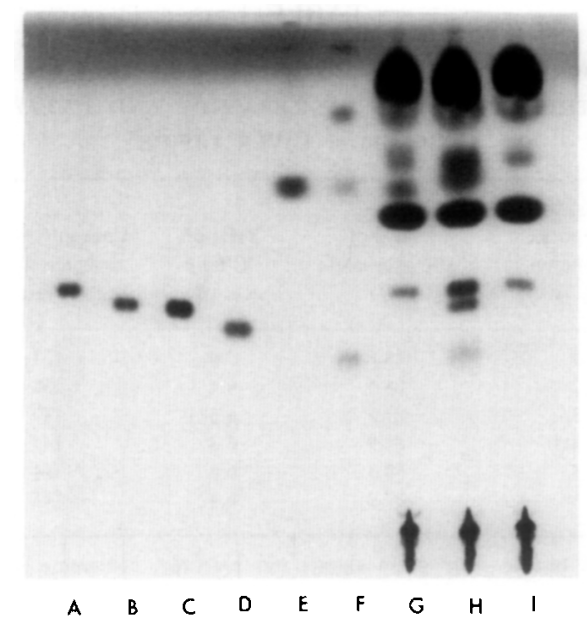

FIG. 1. Thin-layer silica gel plate prepared by the vertical-streak, double-development method. The lanes contain the following: (A) lignoceroyl sphingosine; (B) $4 \mu \mathrm{g}$ of stearoyl sphingosine; (C) $12 \mu \mathrm{g}$ of the same; (D) batyl alcohol; (E) oleic acid; (F) a mixture of glyceryl stearates (the lowest spot is the monoglyceride); (G) low-polarity lipids from rat liver; $(\mathrm{H})$ from calf liver; (I) from mouse liver. The intense large spot near the top of the plate is due to cholesterol esters and the second most intense spot, to cholesterol.

small separation between ceramides and alkyl ethers makes it undesirable to treat the total lipids with alkali to remove polar lipids. Besides, the ceramide esters (22) would then be converted to ceramide and thereby give falsely high analytical values.

The tlc plate showed distinct separation of the shorter- and longer-chain ceramides. Mouse and rat liver ceramides were found to contain mostly long-chain fatty acids, while calf liver and mouse spleen and kidney showed roughly equal amounts of both groups. Brain contained primarily the shorter acids. These observations agree with the analyses of rat brain, liver, and kidney ceramides made by gas-liquid chromatography (4).

The accuracy of our method depends on the purity of the ceramide spot. The uniformity of the spot shape suggested homogeneity, as did a test with a second solvent (chloroform-methanol 90:10) applied in a second dimension. No contaminant could be detected in the ceramide lane, even 
when a sample containing $18 \mu \mathrm{g}$ of ceramide was applied to the origin. (The charring spray readily reveals $0.5 \mu \mathrm{g}$ of lipid.) In addition, comparisons of our data with other data suggest the absence of interfering lipid: we found about $120 \mu \mathrm{g}$ of ceramide per gram of mouse liver (74-day-old animal), while analyses in this laboratory with another, more complex procedure ${ }^{2}$ also yielded values around $120 \mu \mathrm{g} / \mathrm{g}$ for animals of a similar age. Our data for rat liver were $87 \mu \mathrm{g} / \mathrm{g}$ while Iwamori et al. (4) found $76 \mu \mathrm{g} / \mathrm{g}$ for animals of a similar age.

Efforts were made to improve the precision of the sample application step, since this can be one of the major sources of error in tlc methods. It was felt that a small sample volume would be difficult to apply precisely, mainly because of the possibility that an appreciable (and variable) fraction of the sample could end up on the outside of the syringe needle instead of in the tlc powder. Moreover, the use of a small volume would necessitate the use of a small volume to dissolve the sample prior to tlc spotting, increasing the problem of evaporative concentration while handling the tlc syringe.

In order to apply a sample in a relatively large volume, such as $40 \mu \mathrm{l}$, and obtain high resolution with a relatively heavy load, one can either add it to the plate in small portions, as a band at the origin, or in a single vertical streak, as in the method described here. It seemed likely that multiple applications would result in losses on the outside of the syringe needle. We tried using Whatman LK5 and Analtech uniplates, both of which have an inert preadsorbent area below the silica gel layer. This area allows one to apply a large volume in the form of a single vertical streak, as in our procedure, but only a single solvent development step is needed. Unfortunately both plates, unlike the E. Merck plates, revealed a gray background after charring, which yielded a degree of radioactivity quenching that was excessively dependent on the area of pow-

\footnotetext{
${ }^{2}$ Raymond J. Metz and Norman S. Radin, unpublished work.
}

der that was scraped off. In addition, separation between ceramide and cholesterol was poor with our solvent on both plates, and a copper salt precipitated on the surface of the Analtech plate after spraying. An attempt to remove the contaminating material from the Whatman plate by a predevelopment with polar solvent only brought a new impurity out of the preadsorbent region into the adsorbent.

It was found that the size of the ceramide spot depended on the volume of solvent in which it was applied. The degree of quenching obtained when the weight was kept constant $(10 \mu \mathrm{g})$, while the volume of standard solution was varied from 5 to 50 $\mu$, was significantly affected: the observed activities ranged from 3804 to $3546 \mathrm{cpm}$. (The silica gel scraped included the surrounding white background as well as the ceramide spot; the total area was the same for all spots.) For this reason all samples were applied in $40-\mu$ l streaks and volume adjustments to accomodate different ceramide concentrations were made prior to streak application.

\section{Charring and Counting}

Previous publications on the copper/phosphoric spray $(3,18)$ have called for charring at $180^{\circ} \mathrm{C}$. We found that $150^{\circ} \mathrm{C}$ produced distinctly less background color and sensitivity to nonuniformity of spraying. It was important for uniform darkening to avoid the use of a large support block in the oven; apparently uniformity of the heating rate is important. We have observed that lipids of relatively low molecular weight are charred poorly by this spray and believe that evaporation of the compound, together with the water in the sprayed plate, can be a competing phenomenon.

In the previous paper on charring and external standard counting, it was stated that the amount of background silica gel included with the colored band was unimportant (3). In our system, possibly because the samples and spots were smaller, there was a distinct effect of background silica. 
The observed radioactivity was reduced $1.8-2.0 \% / \mathrm{cm}^{2}$ of silica gel. When only the ceramide spots were scraped, without any background material, the standard curve was not linear. Hence all our experiments were carried out by scraping equal areas; this means that heavier spots contained less background.

Tests of the scraping and transfer technique with uncharred radioactive spots showed that there was $99 \%$ of the applied activity in the counting vial. No contamination of the next (nonradioactive) spot could be detected. The glass fiber disks produced slight quenching, less than $1 \%$, but this was constant from vial to vial. Using a larger $\operatorname{disc}(13 \mathrm{~mm})$ produced $4 \%$ quenching, somewhat variable in degree, probably because it did not lie flat at the bottom of the vial.

In the previously described procedure (3), no mention was made of a problem with suspension of the silica gel powder in the counting gel. In our hands, omission of the sonication step yielded a clearly inhomogeneous suspension. Sonication with water alone, at room temperature (more convenient than using a prewarmed bath), yielded a uniform suspension but subsequent shaking with Aquasol produced a nonhomogeneous suspension. Heating the vials after sonication and addition of the scintillation liquid produced a satisfactory suspension, but it seemed more convenient to add both liquids in one step and use hot water in the bath itself.

Trials with several other water-miscible counting solvents produced softer gels, as shown by decreasing amounts of quenching on standing. Presumably the silica gel settled slowly in these mixtures.

In the initial reports $(1,2)$ on the use of scintillation counters as photometers, the light sources were immersed in the colored sample. The inconvenient features of this approach have been noted by Shandon and Nobles (3), who substituted an external radioactive source and a fluorescent gel which also served to suspend the colored powder. This approach should save time, since the external sources used in counters are highly radioactive and need only a minute or so of counting time. However, the use of external standards for determining the quenching in a gelled sample does not always give precise results. With our counter, which uses the $H$ number calculation with an external standard (23), the variability in quench determination seemed excessively large. A Packard Tri-Carb counter, which calculates the channels ratio for the external standard, gave even greater variation.

For the above reasons, we turned to incorporating an internal standard into each sample, using enough tritiated lipid to produce $38,000-25,000$ counts in a 10 -min period. Calibration curves obtained with different amounts of ceramide showed good linearity but the activity observed with an empty tlc lane (no ceramide) was not quite the value extrapolated to zero from the standard curve. Thus one cannot determine the calibration from a single-valued standard and an empty space. A comparison of the four standard ceramide spots on 10 different plates yielded an average correlation

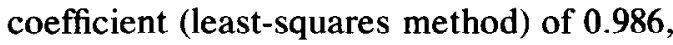
ranging from 0.960 to 0.996 .

The observed counting rate was decreased by about $68 \mathrm{cpm}$ for each microgram of lipid. The standard deviation of a single 25,000 count sample is about 160 counts $(16 \mathrm{cpm})$, equivalent to $0.24 \mu \mathrm{g}$ of ceramide. Thus the variability due to the counting step is relatively low except with small samples, in which case longer counting periods or more radioactive internal standard would be helpful.

Another indication of the reproducibility obtainable with our method was obtained by pipetting out ten $6-\mathrm{ml}$ aliquots of a mouse liver extract, then processing them by the standard method. All 10 samples were applied to a single plate, without standards. The resultant average amounts of radioactivity observed in successive 10-min counting periods were 3070,3030 , and 3027 
cpm and the relative standard deviations were about $1.7 \%$. This degree of precision is quite good for a quantitative tlc method (including the partitioning, transfer, and counting steps).

Tests with different tlc plates yielded a different standard curve for each plate. It is therefore necessary to apply four standard spots (two duplicates) to each plate. This leaves room for six more spots, so three unknown samples can be analyzed in duplicate on each plate. The ceramide weight in each unknown is calculated from the four standard readings with a least-squares regression equation.

\section{Applications of the Method}

The tlc/charring method should be useful for other compounds which yield distinct tlc spots when applied at the level of 2 to 25 $\mu \mathrm{g}$. For the more polar lipids, there could be a problem in the first solvent development step since they might not form a thin band at the solvent front. We have recently found that this problem can be treated (at higher cost) by the use of $E$. Merck tlc plates made with a preadsorbent lower area (No. 11845-9H), which permit use of a single development solvent and give a colorless background on charring.

Our tlc procedure was applied to other mouse tissues from a 31-day-old animal, which were found to contain $93 \mu \mathrm{g} / \mathrm{g}$ brain, $74 \mu \mathrm{g} / \mathrm{g}$ spleen, and $164 \mu \mathrm{g} / \mathrm{g}$ of kidney. Calf liver contained a very high concentration: $0.63 \mathrm{mg} / \mathrm{g}$. It was found that storage of mouse brain-but not liver, kidney, or spleen-at room temperature for even 5 min produced a significant increase in ceramide content. The observed values, derived from groups of five mice each, were $86 \mu \mathrm{g} / \mathrm{g}$ at zero time (quick freezing in pentane held at $-95^{\circ} \mathrm{C}$ ), $132 \mu \mathrm{g} / \mathrm{g}$ at $5 \mathrm{~min}, 156$ at $20 \mathrm{~min}$, and 188 at $30 \mathrm{~min}$.

Since radioactive lipids can be counted on tlc silica gel even after charring (24), the use of our method would require counting half of the lipid spots with water-Aquasol that is free of internal radioactivity.

\section{ACKNOWLEDGMENTS}

This study was supported by Grant NS 03192 from the National Institutes of Health. We are indebted to Inez Mason and Kevin Beyer for technical assistance.

\section{REFERENCES}

1. Snyder, F., and Moehl, A. (1969) Anal. Biochem. 28, 503-509.

2. Noble, R. C., Shand, J. H., and West, I. G. (1979) Lab. Pract. 28, 393-396.

3. Shand, J. H., and Noble, R. C. (1980) Anal. Biochem. 101, 427-434.

4. Iwamori, M., Costello, C., and Moser, H. W. (1979) J. Lipid Res. 20, 86-96.

5. Yahara, S., Moser, H. W., Kolodny, E. H., and Kishimoto, Y. (1980) J. Neurochem. 34, 694699.

6. Iwamori, M., Moser, H. W., McCluer, R. H., and Kishimoto, Y. Biochim. Biophys. Acta 380, 308 -319 .

7. Krivit, W., and Hammarström, S. (1972) J. Lipid Res. 13, 525-530.

8. Samuelsson, K. (1971) Scand. J. Clin. Lab. Invest. 27, 371-380.

9. Karlsson, K.-A., Samuelsson, B. E., and Steen, G. O. (1973) Biochim. Biophys. Acta 316, 317 335.

10. Poulos, A., Hann, C., Phillipou, G., and Pollard, A. C. (1979) Anal. Biochem. 97, 323-327.

11. Bouhours, J. F., and Glickman, R. M. (1976) Biochim. Biophys. Acta 441, 123-133.

12. Tao, R. V. P., Sweeley, C. C., and Jamieson, G. A. (1973) J. Lipid Res. 14, 16-25.

13. Rouser, G., and Yamamoto, A. (1968) Lipids 3, 284-287.

14. Gray, G. M., and Yardley, H. J. (1975) J. Lipid Res. 16, 434-440.

15. Radin, N. S. (1972) in Methods in Enzymology (Ginsburg, V., ed.), Vol. 28, pp. 301-302, Academic Press, New York.

16. Morell, P., Costantino-Ceccarini, E., and Radin, N. S. (1970) Arch. Biochem. Biophys. 141, 738 748.

17. Hara, A., and Radin, N. S. (1978) Anal. Biochem. 90, 420-426.

18. Anderson, L. E., and McClure, W. O. (1973) Anal. Biochem. 51, 173-179.

19. Bowen, D. M., and Radin, N. S. (1969) J. Neurochem. 16, 457-460.

20. Fewster, M. E., Burns, B. J., and Mead, J. F. (1969) I. Chromatogr. 43, 120-126.

21. Platt, S. G. (1978) Anal. Biochem. 91, 357-360.

22. Okabe, H., and Kishimoto, Y. (1977) J. Biol. Chem. 252, 7068-7073.

23. Horrocks, D. L. (1977) Beckman Instruments Publication No. 1095 NUC-77-1T.

24. Noble, R. C., and Shand, J. H. (1980) Lipids 15, 269-271. 